\title{
A high-fat diet alters genome-wide DNA methylation and gene expression in SM/J mice
}

\author{
Madeline Rose Keleher ${ }^{1,4^{*}}$ D, Rabab Zaidi ${ }^{2}$, Lauren Hicks ${ }^{2}$ Shyam Shah ${ }^{2}$, Xiaoyun Xing ${ }^{3}$, Daofeng Li ${ }^{3}$, Ting Wang ${ }^{3}$ and
} James M. Cheverud ${ }^{2}$

\begin{abstract}
Background: While the genetics of obesity has been well defined, the epigenetics of obesity is poorly understood. Here, we used a genome-wide approach to identify genes with differences in both DNA methylation and expression associated with a high-fat diet in mice.

Results: We weaned genetically identical Small (SM/J) mice onto a high-fat or low-fat diet and measured their weights weekly, tested their glucose and insulin tolerance, assessed serum biomarkers, and weighed their organs at necropsy. We measured liver gene expression with RNA-seq (using 21 total libraries, each pooled with 2 mice of the same sex and diet) and DNA methylation with MRE-seq and MeDIP-seq (using 8 total libraries, each pooled with 4 mice of the same sex and diet). There were 4356 genes with expression differences associated with diet, with 184 genes exhibiting a sex-by-diet interaction. Dietary fat dysregulated several pathways, including those involved in cytokine-cytokine receptor interaction, chemokine signaling, and oxidative phosphorylation. Over 7000 genes had differentially methylated regions associated with diet, which occurred in regulatory regions more often than expected by chance. Only $5-10 \%$ of differentially methylated regions occurred in differentially expressed genes, however this was more often than expected by chance $\left(p=2.2 \times 10^{-8}\right)$.

Conclusions: Discovering the gene expression and methylation changes associated with a high-fat diet can help to identify new targets for epigenetic therapies and inform about the physiological changes in obesity. Here, we identified numerous genes with altered expression and methylation that are promising candidates for further study.
\end{abstract}

Keywords: Obesity, Mice, Epigenetics, Methylation, RNA-seq, Gene expression, Diet

\section{Background}

In the US, 35\% of children are overweight and another $26.4 \%$ have obesity [1]. Obesity early in life raises the risk of obesity [2] and liver disease [3] later in life. Today $35 \%$ of adults in the United States are obese, and $42 \%$ are predicted to be by 2030 [4-6]. This is a major threat to public health, since obesity is associated with cancer, stroke, asthma, type 2 diabetes, hypertension, heart attack, and other serious health conditions [7]. The best studied causes of obesity are genetics, the environment, and their interaction [8-13]. The environment changes

\footnotetext{
* Correspondence: madeline.keleher@ucdenver.edu

'Department of Evolution, Ecology, and Population Biology, Washington University in St. Louis, St. Louis, MO 63105, USA

${ }^{4}$ Biology Department, 1032 W. Sheridan Road, Chicago, IL 60660, USA

Full list of author information is available at the end of the article
}

the expression of genes via epigenetic factors such as histone modifications, noncoding RNAs, and DNA methylation, and thus environmental factors causing obesity may do so by inducing epigenetic modifications that change gene expression. Epigenetic variation between individuals may hold the key to more accurate predictions of obesity risk, and better understanding it could lead to new tools for fighting obesity $[14,15]$.

Health problems can result from dysregulated gene expression. While much research has focused on the genetic variants underlying disrupted gene expression in obesity $[12,16]$, far less is known about how diet changes gene expression through epigenetics to cause obesity. Technological advances have made epigenetic studies more feasible, and new journals and scientific meetings

(c) The Author(s). 2018 Open Access This article is distributed under the terms of the Creative Commons Attribution 4.0 International License (http://creativecommons.org/licenses/by/4.0/), which permits unrestricted use, distribution, and reproduction in any medium, provided you give appropriate credit to the original author(s) and the source, provide a link to the Creative Commons license, and indicate if changes were made. The Creative Commons Public Domain Dedication waiver (http://creativecommons.org/publicdomain/zero/1.0/) applies to the data made available in this article, unless otherwise stated. 
have been created to address the explosion of epigenetics research [17]. The best-characterized epigenetic mechanism is DNA methylation; however, even this is not well-understood. When the majority of the cytosines in a promoter region are methylated, gene expression tends to be lower than when these regions are hypomethylated [18]. This is not always the case, however, and methylation at other regulatory regions can actually increase expression [19]. To fill in the gaps of our understanding of epigenetics, it is important to explore the methylation profile of not just promoter regions in candidate genes but of the entire genome, as we do here.

Changes in DNA methylation do not always imply changes in gene expression, or vice versa. For instance, rats fed a diet high in fat and sugar had higher hepatic expression of the Hadhb gene, but they had no corresponding changes in methylation [20]. Furthermore, when Rönn et al. [21] analyzed DNA methylation in men's adipose tissue before and after 6 months of exercise, they found methylation changes in 7663 genes, but only 197 of those genes also had expression changes. This illustrates a common finding in obesity epigenetics studies: while there are methylation differences associated with obesity, many changes in DNA methylation do not cause detectable changes in the expression of nearby genes. More research needs to be done to characterize the relevant DNA methylation changes in obesity. So far, candidate-gene studies have revealed DNA methylation differences between obese and lean individuals in a handful of genes in different tissues [22-25]. Genome-wide methylation studies have revealed differentially methylated regions in genes involved in cell differentiation, the immune system, and transcriptional regulation [23]. To understand how changes in DNA methylation affect gene expression in obesity, however, it is important to consider genetic background. C57BL/6 is a widely studied mouse strain that has contributed enormously to our understanding of obesity, however its response to a high-fat diet differs from that of other mouse strains. Compared to C57BL/6, when fed a high-fat diet BALB/c mice have been shown to have some degree of metabolic protection [26], DBA/2 mice are $10 \%$ heavier and have decreased mean pancreatic islet area [27], $129 \mathrm{~T} 2$ mice have higher glucose levels following a glucose injection [27], and though both C57BL/6 and BFMI mic have reduced DNA methylation of the $M c 4 r$ gene, only BMFI mice have increased $M c 4 r$ expression [28]. Genetic background clearly affects the response to dietary fat, and thus it is important to determine if findings from the most commonly studied mouse strains are replicated in other strains before expecting the research to be informative for human health. Here, we investigated obesity epigenetics the Small $(\mathrm{SM} / \mathrm{J})$ strain of mouse, a strain that is less commonly studied overall but that our lab group has extensively characterized in the context of quantitative genetics, gene expression, and its obesogenic response to dietary fat [29-31].

DNA methylation is just one of several regulatory factors that control gene expression, thus we did not expect to find methylation changes in all differentially expressed genes. However, we were interested in genes where expression differences and methylation differences coincided, as these could make promising candidate genes for epigenetic therapies in the future. We tested the hypothesis that a high-fat diet would alter the expression and methylation of genes involved in obesity and diabetes. Additionally, we tested the hypothesis that there would be sex differences in the genes affected by a high-fat diet.

\section{Materials and methods Animal rearing}

To investigate how a high-fat diet alters gene expression and methylation, we studied the inbred Small (SM/J) mouse strain from The Jackson Laboratory (Bar Harbor, Maine). The SM/J strain originated from a selective breeding experiment for small size at 60 days of age [32]. In response to the same high-fat diet as used in the present study we have previously shown that SM/J mice have reduced glucose tolerance [30], increased organ weights [30], and have 2137 differentially expressed transcripts in the liver as assessed by an Illumina ${ }^{\circ}$ WG- 6 v. 2 BeadChip [31]. Fifteen males and 15 females born at Loyola University in Chicago were bred to produce 56 mice for the study population. The offspring were weaned onto a low-fat (LF) or high-fat (HF) diet at 3 weeks of age (16 HF females, 12 LF females, 18 HF males, and $10 \mathrm{LF}$ males). The diets were designed to be as similar as possible in terms of nutrients and calories; however, $15 \%$ of the calories came from fat in the LF diet (Research Diets D12284), whereas $42 \%$ did in the HF diet (Harlan Teklad diet TD.88137) (Additional file 1: Table S1). Most of the fat in the LF diet came from polyunsaturated fat, whereas most of the fat in the HF diet came from saturated fat, which has been shown to increase expression of genes involved in inflammation and lipogenesis in mouse livers [33]. Previous work by Erich et al. [30] showed that SM/J mice consume the same amount of food whether they are on the HF diet or the LF diet. The mice were fed ad libitum and after weaning each mouse was housed with one other mouse of the same sex and diet in a cage containing a wooden gnawing block (Bio Serve), a red privacy hut (Alt Design), and a $2 " \times 2 "$ cotton nestlet (Ancare). Procedures followed an approved Institutional Animal Care and Use Committee protocol (Project \#1188, Loyola University). 


\section{Obesity phenotypes}

The mice were weighed weekly from 1 to 17 weeks of age. They underwent an intraperitoneal glucose tolerance test (IPGTT) at 15 weeks of age. All tests started with a 4-h fast at 6:00 am, followed by a tail snip to measure the baseline glucose level, and an intraperitoneal injection of glucose $(1 \mathrm{mg} / \mathrm{g}$ body weight). Glucose measurements were then taken from tail blood at 30, 60, and 120 min after injection. At 16 weeks of age, the mice received an intraperitoneal insulin tolerance test (IPITT), with the same protocol as the IPGTT except that insulin was injected instead of glucose $(0.75 \mathrm{mU}$ insulin/g body weight). For both tests, the glucose values at the 4 different time points were used to calculate the area under the curve (AUC) using the trapezoidal summation method [34].

At 17 weeks of age, the mice were fasted for $4 \mathrm{~h}$ and sacrificed via carbon dioxide asphyxiation between 10:00 am and 2:00 pm. Blood from a cardiac puncture $(0.5 \mathrm{~mL})$ was centrifuged at $4{ }^{\circ} \mathrm{C}$, and serum levels of insulin, leptin, triglycerides, glucose, cholesterol, and free fatty acids were measured. We performed the necropsies on ice and recorded the weights of the liver, heart, reproductive fat pad, kidneys, spleen, brown fat, and skeletal muscle (gastrocnemius). We weighed only the reproductive fat pad rather than all of the fat pads, because it is strongly genetically $\left(\mathrm{h}^{2}=0.7-0.9\right)$ and phenotypically correlated $(r=0.67-0.82)$ with the other fat pads $[9,35]$. We flash-froze liver tissue in liquid nitrogen for RNA extraction.

We performed multivariate analysis of variance (MANOVA) using the stats package in $\mathrm{R}$ to analyze differences in the obesity traits. Multivariate tests were performed on the following three groups of traits: weekly weights, diabetes-related traits (week 15 and 16 weights, baseline glucose during the IPGTT, IPGTT AUC, baseline glucose during the IPITT, and IPITT AUC), and necropsy traits (week 17 weight, organ weights, and serum biomarkers), as well as all the associated univariate tests (ANOVA). Differences were interpreted as significant for $p$-values less than 0.05 .

\section{Gene expression}

We extracted RNA from the liver tissue using the Qiagen RNeasy Plus Mini kit for RNA-seq with poly-A selection. A total of 21 libraries were sequenced, each with 2 mice of the same sex and diet pooled together. There were $6 \mathrm{LF}$ female libraries, and 5 of each of the other sex-diet groups. A $1 \times 50$ single read sequencing run was done on an Illumina HiSeq 2500 machine (Illumina Inc.). The FastQ files were aligned to the Ensembl release 76 assembly using STAR version 2.0.4b [36]. The gene counts were then analyzed with the $R$ package edge $R$ [37]; differences in library size were accounted for with a TMM normalization, and genes with counts of zero were filtered out. The weighted likelihoods were then calculated using the voom function in the $\mathrm{R}$ package Limma based on the mean-variance relationship of each gene and transcript. Generalized linear models were used to test for differential expression. We tested for the effect of sex, of diet, and of a sex*diet interaction on gene expression. Any gene with a false discovery rate (FDR) adjusted q-value of 0.05 or less was considered differentially expressed. We performed a pathway analysis using the $\mathrm{R}$ package GAGE [38] to identify pathways that were significantly up-regulated, down-regulated, or perturbed in both directions. We visualized the pathways with the R package Pathview [39].

We validated the differential expression for 3 genes in the females (Adam11, Lad1, and Galnt10) and 3 in the males (Adam11, Abcg8, and Col1a1) using rt-qPCR, with Gapdh as a normalizer (Additional file 1: Table S2). To do this, we extracted total RNA from the livers of $3 \mathrm{HF}$ and 3 LF mice of each sex using Tri-Reagent (MRC), following the manufacturer's instructions. The concentration and quality of the RNA from each sample was assessed twice with a NanoDrop Spectrophotometer, and only samples with a $260 / 280$ ratio between $1.7-2.1$ and a 260/230 ratio between 2.0-2.4 were used. We then immediately reverse transcribed the RNA to cDNA using the High-Capacity cDNA Reverse Transcription Kit (Applied Biosystems), following the manufacturer's instructions. Primers were selected from the literature, and if none were found we used PrimerBank to design the primers. All primers were synthesized by Thermo Fisher Scientific (the sequences are listed in Additional file 1: Table S3). We performed RT-qPCR using $10 \mu \mathrm{L}$ of PowerUp ${ }^{\text {m }}$ SYBR $^{\circ}$ Green Master Mix (Thermo Fisher), $1 \mu \mathrm{L}$ of the forward primer, $1 \mu \mathrm{L}$ of the reverse primer, $4 \mu \mathrm{L}$ of 20 -fold diluted $\mathrm{cDNA}$, and $4 \mu \mathrm{L}$ of water, with a total volume of $20 \mu \mathrm{L}$ for each reaction. The RT-qPCR was performed with a StepOnePlus Real-Time PCR System (Applied Biosystems) at the following conditions: $20 \mathrm{~s}$ at $95^{\circ} \mathrm{C}$, followed by 40 cycles of $3 \mathrm{~s}$ at $95^{\circ} \mathrm{C}$ and $30 \mathrm{~s}$ at $60^{\circ} \mathrm{C}$. For each of the 3 biological replicates, 3 technical replicates were used, along with a no-template control and a no-reverse-transcriptase control. We did a relative quantification of each gene using Gapdh as a reference using the comparative $\Delta \Delta \mathrm{Ct}$ method. There were no differences in Gapdh expression between the diet treatment groups. The expression data is available on NCBI's Gene Expression Omnibus (GEO), record number GSE121525.

\section{DNA methylation}

We performed a phenol-chloroform extraction to isolate DNA from the liver tissue. Genome-wide DNA methylation was then assessed with Methylated DNA 
Immunoprecipitation Sequencing (MeDIP-seq) and Methylation-sensitive Restriction Enzyme Sequencing (MRE-seq), as described by Li et al. [40]. MeDIP-seq detects methylated sites while MRE-seq detects unmethylated sites, and when used in combination these two techniques provide a single CpG resolution methylation map that has high concordance with whole-genome bisulfite sequencing at only a fraction of the cost [41]. The sequencing was done by running $2 \times$ 75 bp paired-end reads via Illumina NextSeq 500. Four mice of the same sex and diet treatment were pooled per library, yielding 2 biological replicates per group. The NIH Epigenomics Project recommends using 2 replicates with a combined total coverage of 30x for whole genome bisulfite sequencing [46], although this can quickly become cost prohibitive for larger sample sizes. When MRE-seq and MeDIP-seq are combined they have comparable coverage to whole genome bisulfite sequencing [39], making 2 replicates sufficient here.

To synthesize the MRE-seq and MeDIP-seq data and test for differential methylation, we used the $\mathrm{R}$ package methylMnM, which was specifically designed for this purpose. First, we split the mouse $\mathrm{mm} 9$ genome into 500-base-pair windows (for a total of 5,283,825 windows); then, we assessed the proportion of methylated CpGs in each window; and from there we calculated the novel M\&M test statistic to determine if the methylation level was different between the two diet treatments [42]. M\&M tests two groups at a time, which yielded 4 pairwise comparisons of the female libraries and 4 of the males. To synthesize the information from all 4 library comparisons per sex, we used Fisher's combined probability test [43]. To examine DMRs due to diet in the females, the $p$-value from the $M \& M$ test comparing the first HF-female library with the first LF-female library was combined with the $\mathrm{p}$-value from the $M \& M$ test comparing the second HF-female library with the second LF-female library according to the following equation by Fisher:

$$
X_{2 k}^{2} \sim-2 \sum_{i=1}^{k} \ln \left(p_{i}\right)
$$

In this case, $p_{i}$ is the $p$-value from the pairwise $M \& M$ test, and $\mathrm{k}$ is the number of tests combined. We calculated a combined $p$-value for each 500-base-pair window, corrected for FDR with the Benjamini-Hochberg method [44], and calculated how many of these windows were differentially methylated based on q-value cutoffs of less than 0.05 and 0.01 .

For each DMR, we then identified the nearest gene to it, if it fell within a gene, if it fell within a promoter, if it contained a known regulatory element listed in Ensembl (mouse genome assembly GRCm38.p5) [45], and if the gene closest to it was already known to be involved in obesity, diabetes mellitus, or cardiovascular diseases based on Phenopedia's continuously updated list of genes uncovered by genetic association studies in humans (downloaded May 7, 2017). We also classified the DMR as being either in an intergenic region, exon, intron, or promoter. This was done using the full list of introns, exons, and genes downloaded from the $\mathrm{NCBI} 37 / \mathrm{mm} 9$ assembly on the UCSC Genome Browser. If a DMR overlapped both an intron and an exon, it was classified as falling within an exon. It was classified as a promoter if it was within 2000 base pairs upstream of a transcription start site or 600 base pairs downstream of one. To determine if the DMRs were associated with gene expression, we randomized the DMRs across the genome and calculated how many fell within differentially expressed genes due to chance. To account for the general underrepresentation of DMRs in intergenic regions during the randomization, the percent of DMRs that were allowed to be randomized into intergenic regions was equal to the percent that actually exist in those regions. We performed a chi-square test to determine if the DMRs were found in regulatory regions at a greater rate than expected due to chance.

Prior to combining the $p$-values with Fisher's combined probability test, we compared the replicates, which represents technical and biological noise. There was one significant DMR when comparing the two LF male libraries $(\mathrm{q}<0.05)$ and 21 significant DMRs when comparing the HF male libraries, whereas each comparison of an LF with an HF male library had between 22 and 66 DMRs. There were 84 significant DMRs when comparing the two LF female libraries with each other and 22 significant DMRs when comparing the HF female libraries, whereas each comparison of an LF with an HF female library had between 9 and 190 DMRs. Very few DMRs were found across multiple comparisons. To understand the meaningful methylation differences, it is important to use replicates, as we did here. The methylation data is available on NCBI's Gene Expression Omnibus (GEO), record number GSE122016.

\section{Results}

\section{Obesity phenotype}

Diet significantly affected the weekly weights $(p=1.59 \times$ $\left.10^{-7}\right)$, diabetes-related traits $\left(p=8.11 \times 10^{-11}\right)$, and necropsy traits $\left(p=6.21 \times 10^{-7}\right)$ (Additional file 1: Table S4). After only one week of being on the diet treatment (4 weeks of age), high-fat (HF) mice weighed significantly more than low-fat (LF) mice, and the difference became more pronounced with age (Fig. 1). The HF mice had reproductive fat pads that were more than 8 times larger than the LF mice. The ANOVA revealed an overall increase in all organ weights on 


\section{Weekly Weights}

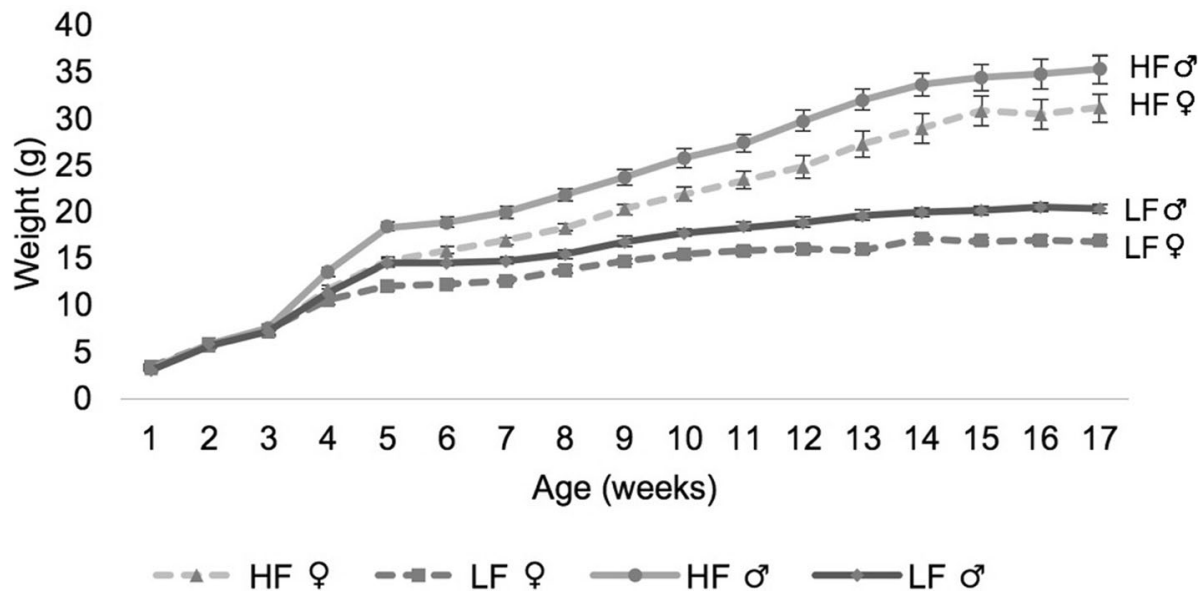

Fig. 1 The average weight of mice in grams ( \pm one standard error) from 1 to 17 weeks of age. Diet had a statistically significant effect from 4 weeks of age and on. HF $=$ High-fat diet, and LF $=$ Low-fat diet. LF male $n=10$, HF male $n=18$, LF female $n=12$, HF female $n=16$

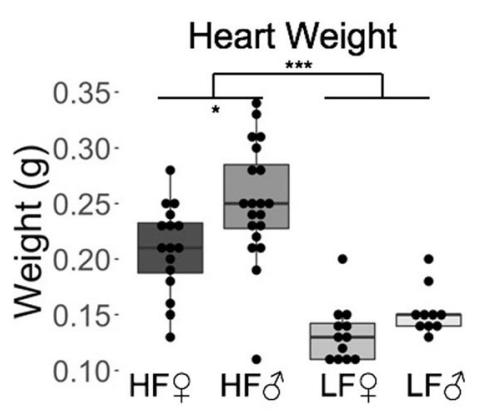

Fat Pad Weight
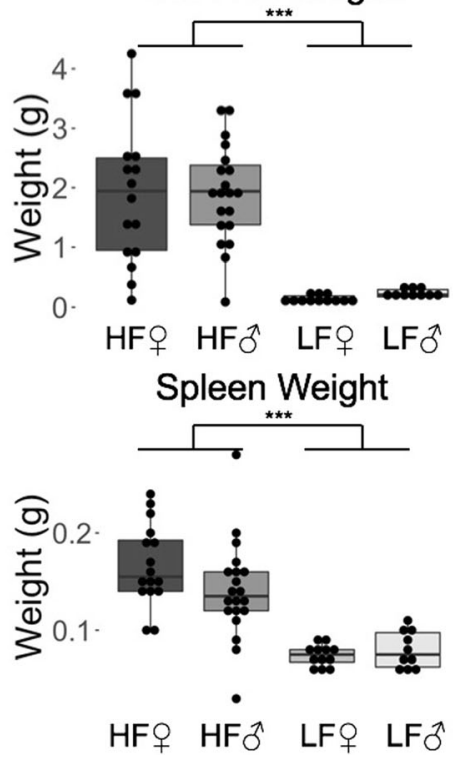

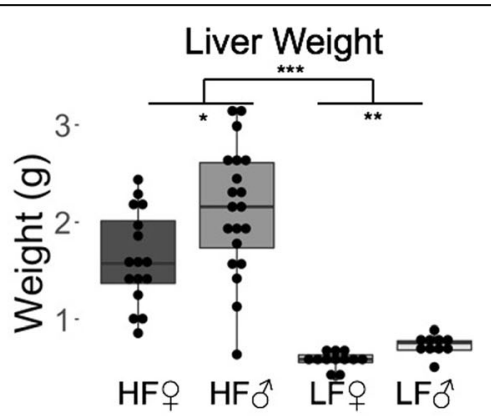

Avg Kidney Weight
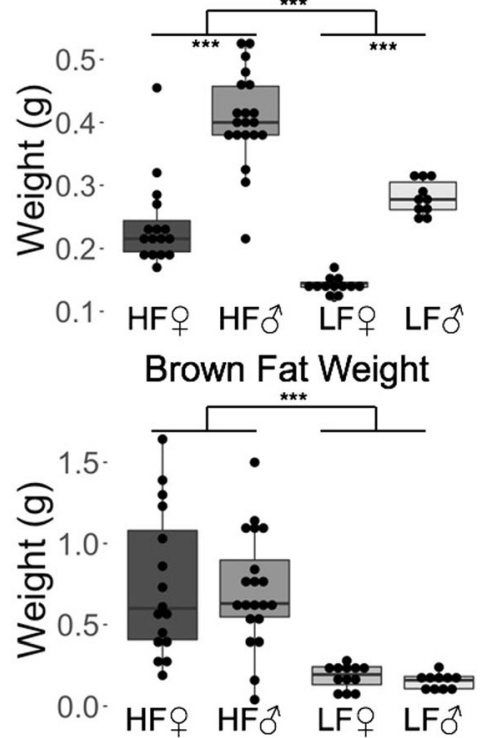

Fig. 2 The average organ weights for each sex and diet group in grams. All organ weights were significantly heavier in the high-fat mice than the low-fat mice. The liver, heart, and kidneys were heavier in males than females. $H F=$ High-fat diet, and $L F=L$ Low-fat diet. $L F$ male $n=10, H F$ male $n=18$, LF female $n=12$, HF female $n=16 .{ }^{* * *} p<0.001,{ }^{* *} p<0.01,{ }^{*} p<0.05$, absence of asterisks indicates not-significant 
an HF diet, including 2.8 times heavier livers $(1.51 \times$ $\left.10^{-13}\right)$ and 1.6 times heavier hearts $\left(2.39 \times 10^{-8}\right)$ (Fig. 2, Additional file 1: Table S4).

Diet also significantly affected the response to intraperitoneal glucose and insulin tolerance testing, with $\mathrm{HF}$ mice having higher glucose area under the curve (AUC) values for both the IPGTT $\left(p=5.16 \times 10^{-7}\right)$ (Fig. 3) and the IPITT $\left(p=2.53 \times 10^{-8}\right)$ (Fig. 4), indicating impaired glucose and insulin signaling. Figures 3 and 4 depict curves of the mouse that had the median AUC value per sex and diet group to illustrate what the curves looked like. All serum biomarkers except for free fatty acids had higher levels due to an HF diet (Fig. 5), particularly in males.

HF mice had higher levels of cholesterol $(p=5.05 \times$ $10^{-12} ; 2.2$ times higher in females, 3 times higher in males), triglycerides $(p=0.003 ; 1.3$ times higher in females, 2.1 times higher in males), glucose $(p=1.35 \times$ $10^{-4}$; 1.4 times higher in females, 2 times higher in males), and insulin ( $p=1.05 \times 10^{-4} ; 6.7$ times higher in females, 38 times higher in males) (Fig. 5). HF mice also had substantially higher levels of leptin $\left(p=7.61 \times 10^{-8}\right)$, with HF female mice having 20 times more leptin in their serum and HF male mice having 42 times more than LF mice (Fig. 6a). The correlation between serum leptin levels and the leptin receptor (Lepr) gene was negative $(R=-0.481, p=0.027)$ (Fig. $6 \mathrm{~b})$, with HF mice having 7 times lower expression of Lepr in the liver than LF mice (Fig. 6c). Leptin had a strong positive correlation with fat pad weight $(R=0.917, p=5.262 \times$ $10^{-8}$ ) (Fig. 6d).

The body weights $\left(p=4.17 \times 10^{-5}\right)$, diabetes traits $(p=$ $0.002)$, and serum biomarkers $\left(p=1.54 \times 10^{-14}\right)$ were also significantly affected by sex. Irrespective of diet, males weighed more $\left(p=4.64 \times 10^{-4}\right.$ at 17 weeks $)$, had heavier livers $(p=0.014)$ and kidneys $\left(p=2.20 \times 10^{-16}\right)$, and had higher glucose AUC values during glucose tolerance testing $\left(p=1.59 \times 10^{-5}\right)$.

Although the sex-by-diet interaction effect was not significant on a multivariate level, it was significant on a univariate level for cholesterol $(p=0.005)$, insulin $(p=$ 0.002 ), and glucose AUC during the intraperitoneal insulin tolerance test (IPITT) $(p=0.024)$.

\section{Gene expression}

The multidimensional scaling (MDS) plot indicated that the gene expression libraries clustered primarily by sex (dimension 1, 74\% of the variance) and then by diet (dimension 2) (Additional file 1: Figure S1). Diet altered the expression of 4356 genes in the liver $(\mathrm{q}<0.05)$, or

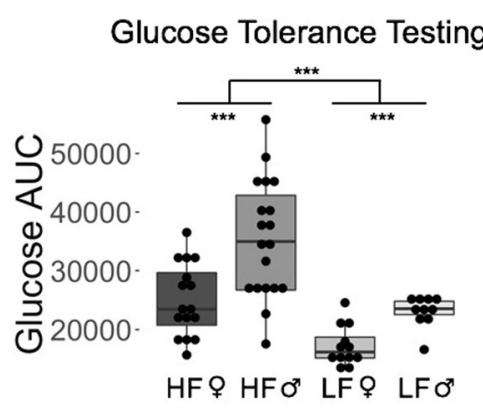

GTT Curve: HF 9

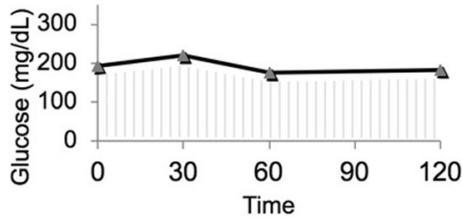

GTT Curve: HF ơ

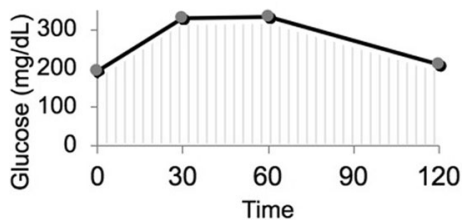

GTT Curve: LF o

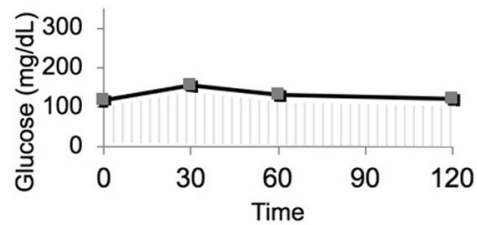

GTT Curve: LF ơ

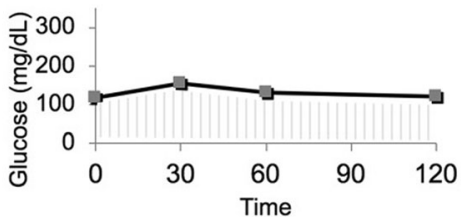

Fig. 3 High-fat diet mice had an elevated response to glucose tolerance testing. HF = High-fat diet, and LF = Low-fat diet. LF male $n=$ 10, HF male $n=18$, LF female $n=12$, HF female $n=16$. ${ }^{* * *} p<0.001,{ }^{* *} p<0.01,{ }^{*} p<0.05$, absence of asterisks indicates not-significant 


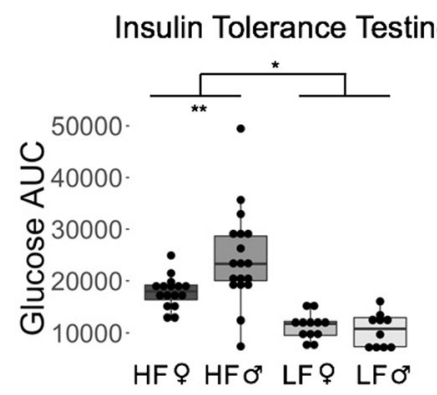

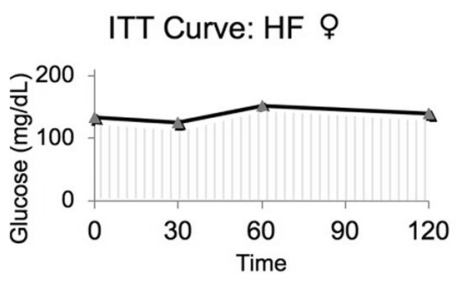

ITT Curve: HF ơ

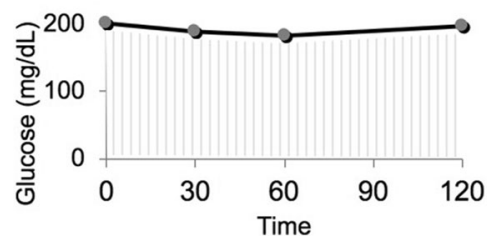

ITT Curve: LF \&

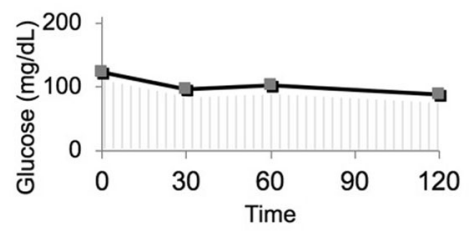

ITT Curve: LF Ơ

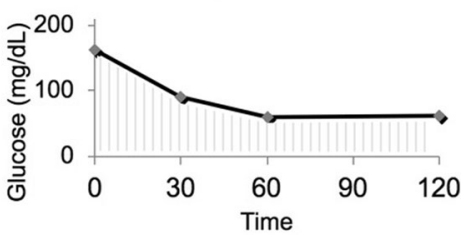

Fig. 4 High-fat diet mice had a reduced sensitivity to insulin tolerance testing. $H F=$ High-fat diet, and $L F=L$ Low-fat diet. $L F$ male $n=10$, HF male $n=18$, LF female $n=12$, HF female $n=16 .{ }^{* *} p<0.001,{ }^{* *} p<0.01,{ }^{*} p<0.05$, absence of asterisks indicates not-significant
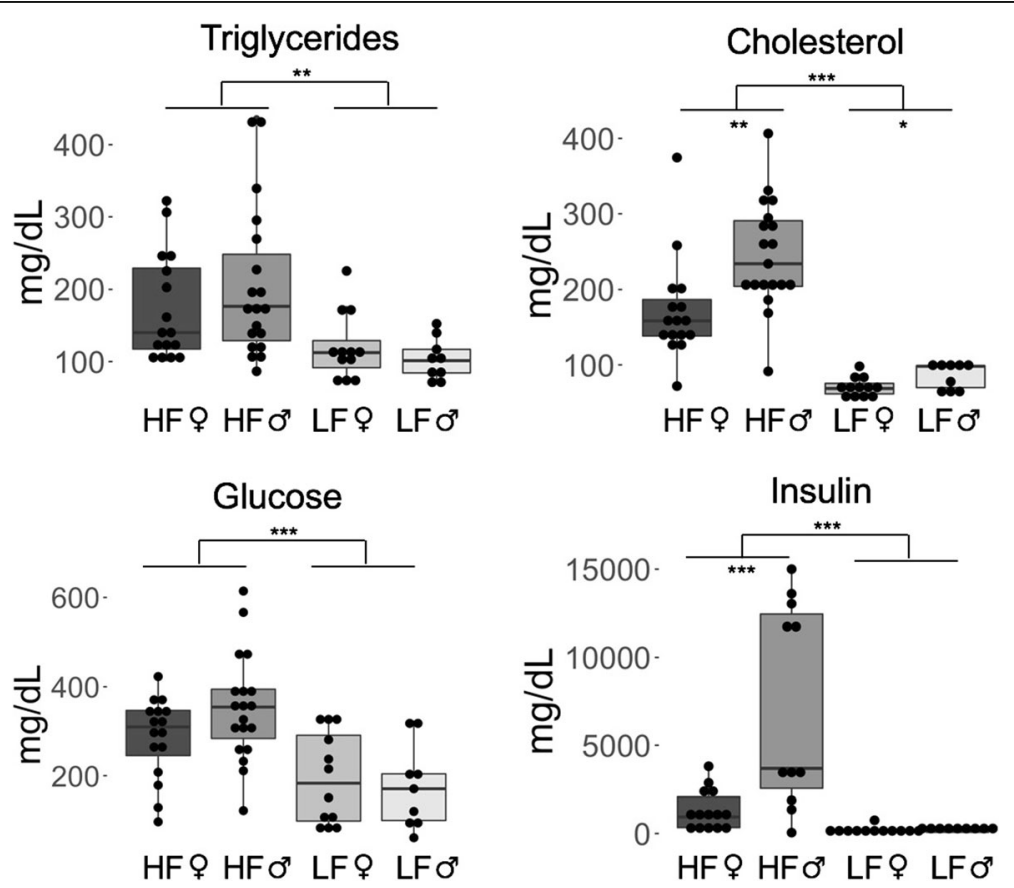

Fig. 5 High-fat diet mice had higher levels of triglycerides, cholesterol, glucose, and insulin in their serum than low-fat diet mice. HF = High-fat diet, and LF $=$ Low-fat diet. ${ }^{* *} p<0.001,{ }^{* *} p<0.01,{ }^{*} p<0.05$, absence of asterisks indicates not-significant 

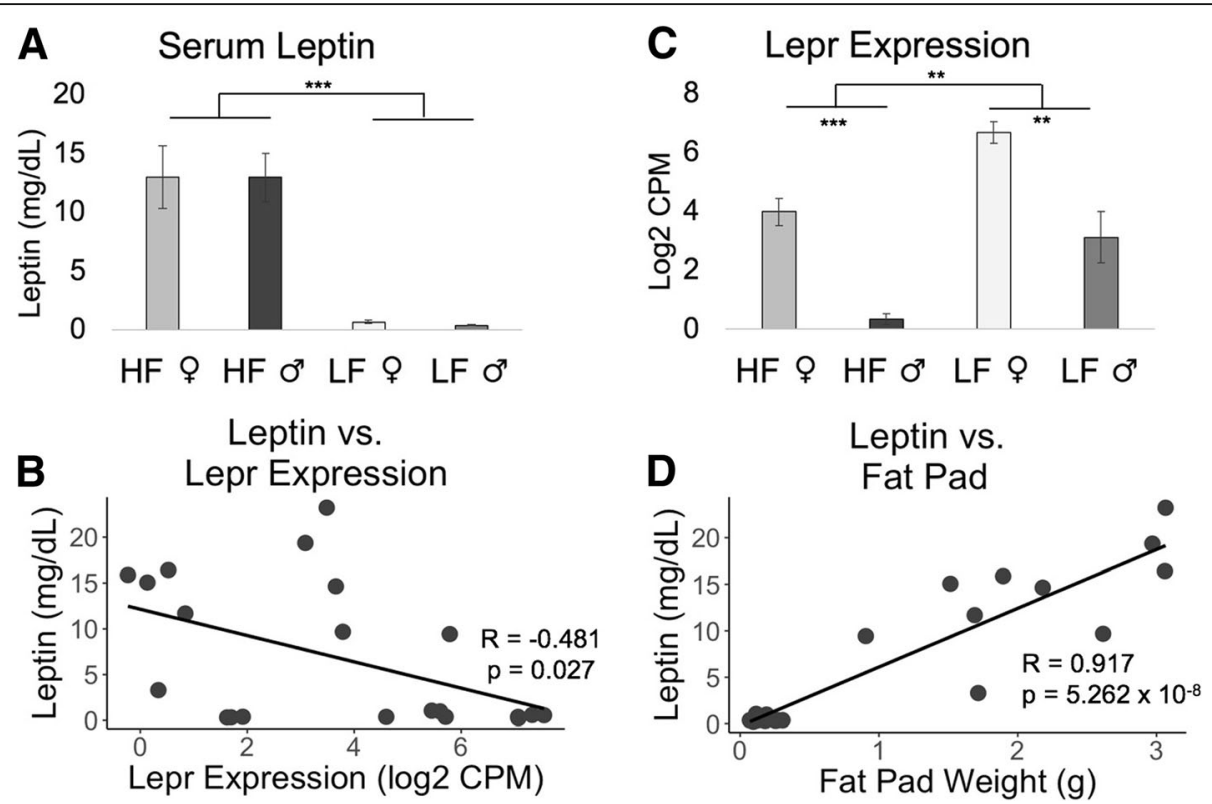

Fig. 6 a A high-fat diet drastically increased leptin levels, $\mathbf{b}$ and was negatively correlated with hepatic expression of the leptin receptor Lepr gene. $\mathbf{c}$ High-fat-fed mice had signifcantly reduced expression of Lepr. $\mathbf{d}$ Serum leptin levels were positvely correlated with the weight of the reproductice fat pad. $\mathrm{HF}=$ High-fat diet, and $\mathrm{LF}=\mathrm{Low}$-fat diet. Error bars represent \pm a single standard error. ${ }^{* * *} p<0.001,{ }^{* *} p<0.01,{ }^{*} p<0.05$, absence of asterisks indicates not-significant

approximately one-fifth of the genome. The $\log 2$ fold changes of the significantly differentially expressed genes ranged from -0.19 to -5.83 and 0.20 to 7.17 . More differentially expressed genes were detected in males (3330) than in females (1750). Of the genes that were differentially expressed, 848 were differentially expressed due to diet only in females and 2428 were unique to males (Additional file 1: Tables S5 and S6). There were 184 genes with significantly different expression due to a sex-by-diet interaction, which a GO Enrichment analysis (Gene Ontology Consortium) showed were enriched for three biological processes: epoxygenase P450 pathway $\left(p=2.36 \times 10^{-5}\right)$, oxidation-reduction process $\left(p=5.58 \times 10^{-5}\right)$, and response to stilbenoid $\left(p=5.21 \times 10^{-3}\right)$.

The GAGE pathway analysis revealed that an HF diet changed the regulation of 7 pathways (Additional file 1: Table S7). This included the downregulation of the oxidative phosphorylation pathway and upregulation of the cytokine-cytokine pathway, indicating that the HF diet reduced mitochondrial function and increased inflammation (Additional file 1: Figure S2). In females, there were 4 pathways upregulated by an HF diet: cytokine-cytokine receptor interaction, chemokine signaling, cell adhesion molecules, and the natural killer cell mediated cytotoxicity pathways. In males, the cytokine-cytokine receptor interaction pathway was also upregulated by an HF diet, while the ribosome and oxidative phosphorylation pathways were downregulated
(Additional file 1: Table S8). In females, 29 GO Biological Processes were upregulated, nearly all of them related to the immune system. Even more were upregulated in males, with 61 affected processes, again mostly involved in the immune system (Additional file 1: Table S9). No GO processes were downregulated, which was perhaps related to the strong upregulation of inflammation.

\section{Methylation}

A q-value cutoff of 0.05 revealed tens of thousands of differentially methylated regions (DMRs) associated with diet, which encompassed $0.6-0.8 \%$ of the nearly 5.3 million 500-base-pair windows in the genome. A cutoff of 0.01 was more discriminating, with less than $0.04 \%$ of windows falling below it, allowing us to focus on a few thousand genes with differential methylation (Additional file 1: Tables S10 and S11). The comparison of HF and LF females resulted in 2356 DMRs $(\mathrm{q}<0.01)$, which was more than the 1539 DMRs between the HF and LF males (Additional file 1: Table S12). There were even more DMRs due to sex than diet, with HF males and females differing at 3831 regions and LF males and females differing at 5632 regions $(\mathrm{q}<0.01)$. A greater percentage of DMRs were found on the $\mathrm{X}$ chromosome in the between-sex comparisons $(2.3-2.8 \%)$ than in the within-sex comparisons $(0.1-0.3 \%, \mathrm{q}<0.01)$.

In all, 7814 genes (38.3\% of genes) in the liver contained at least one diet-induced DMR $(\mathrm{q}<0.05)$ between its outermost transcription start and end sites in the 

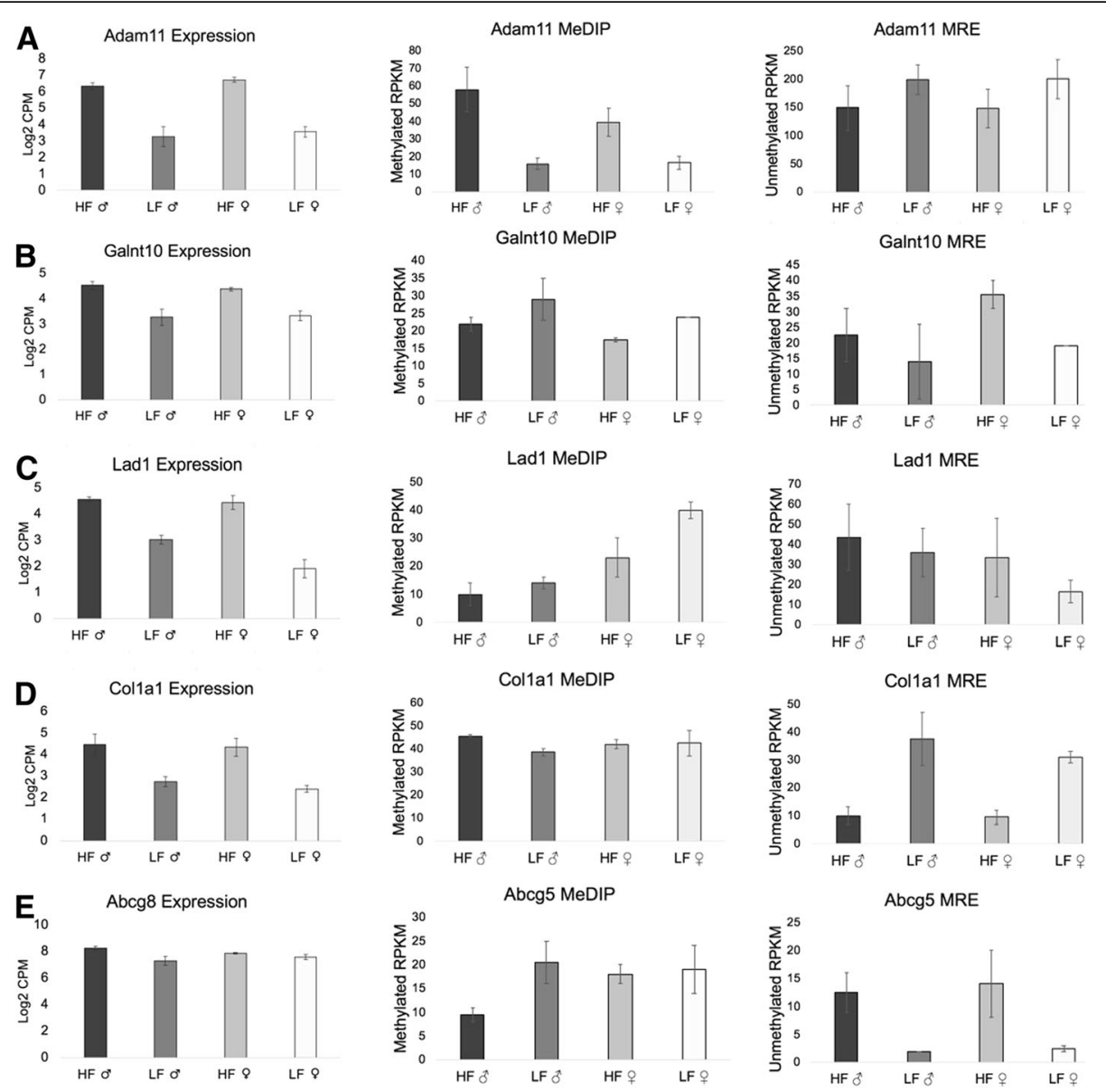

Fig. 7 a High-fat (HF) diet mice had significantly higher methylation and higher Adam 11 expression than low-fat (LF) diet mice. $\mathbf{b}$ HF mice also had higher Galnt10 gene expression, with HF females having significantly less methylation in their first intron than LF females. $\mathbf{c}$ HF mice had higher expression of Lad1, and the first intron (also a promoter region) had lower methylation in HF females than LF females. $\mathbf{d}$ HF mice had higher expression of the Colla1 gene than LF mice, with significantly increased methylation in HF males across exons 23 and 24 compared to LF males. e HF males had less methylation at a region between the genes Abcg8 and Abcg5, which lie head-to-head, and the HF males had significantly increased expression of both Abcg8 and Abcg5

females, as did 7086 genes (34.7\%) in the males (Additional file 1: Table S13). When the DMRs were assigned to one of four categories, $15 \%$ fell within promoters, $25 \%$ in exons, $34 \%$ in introns, and $31 \%$ were in intergenic regions. Not all of these categories were mutually exclusive, since several DMRs encompassed both exons and promoters. Many of the DMRs were in regulatory regions, including $10-12 \%$ in enhancers, $14-16 \%$ in promoters, $3-7 \%$ in CTCF transcription factor binding sites, and $34 \%$ in promoter flanking regions as defined by Ensembl (Table 1). Across the genome, only $4 \%$ of the windows overlapped enhancers, $2 \%$ overlapped CTCF binding sites, and $8 \%$ overlapped promoter flanking regions. DMRs were far more likely to be found in these regulatory regions than in non-regulatory regions of the genome $\left(x^{2}, p<1 \times 10^{-10}\right)$.

Although only a small percentage of the DMRs fell in differentially expressed genes, it nevertheless happened
Table 1 The distribution of significant differentially methylated regions (DMRs) $(q<0.01)$ across the genome associated with diet. Numbers indicate how many 500 base pair windows overlap each genomic region, with the percent of the total significant DMRs that overlap such regions in parentheses for the female and male mice. The percent of windows across the entire genome that overlap these genomic regions is listed as a comparison, illustrating the overrepresentation of regulatory regions in the DMRs

\begin{tabular}{llll}
\hline Region & Female DMRs & Male DMRs & Whole Genome \\
\hline Enhancer & $237(10.0 \%)$ & $180(11.7 \%)$ & $3.5 \%$ \\
CTCF Binding Site & $157(6.7 \%)$ & $55(3.6 \%)$ & $1.7 \%$ \\
TF binding site & $33(1.4 \%)$ & $12(0.8 \%)$ & $0.3 \%$ \\
Promoter Flanking Region & $795(33.8 \%)$ & $522(33.9 \%)$ & $8.1 \%$ \\
Promoter & $370(15.7 \%)$ & $215(14.0 \%)$ & $4.5 \%$ \\
Exon & $598(25.4 \%)$ & $415(27.0 \%)$ & $7.5 \%$ \\
Intergenic & $748(31.8 \%)$ & $471(30.6 \%)$ & $58.6 \%$ \\
\hline
\end{tabular}


more often than expected by chance $\left(\chi^{2}, p=2.2 \times 10^{-8}\right)$. In the females, 2170 (5.6\% of) DMRs fell within differentially expressed genes, whereas only 1994 (5.1\%) were expected to by chance. In the males, 3209 (10.2\% of) DMRs fell within differentially expressed genes, whereas only $2992(9.5 \%)$ were expected to by chance.

\section{Candidate genes with differential methylation and differential expression}

The differentially expressed gene with the lowest q-value in the females was ADAM metallopeptidase domain 11 $\left(\right.$ Adam11) $\left(\mathrm{q}=7.6 \times 10^{-20}\right)$, which also had three adjacent DMRs encompassing exons $14-18$ of the gene. The males had the same DMRs and exhibited the same pattern as the females of having higher methylation and higher Adam11 expression due to an HF diet (Fig. 7a). Although the Adam11 gene is known to be expressed in the mouse liver (MGI Gene Expression Database), its role in obesity and diabetes has not been discussed. It belongs to a family of genes involved in cell signaling, migration, and adhesion, and mice lacking Adam11 have impaired spatial learning and motor coordination, along with a reduced response to inflammatory pain [47]. Perhaps the increased inflammation associated with obesity leads to an increase in inflammation-related pain.

An HF diet also increased expression of the UDP-N-acetyl-alpha-D-galactosamine:polypeptide N-acetylgalactosaminyltransferase 10 (Galnt10) gene in both the males and the females. A DMR in its first intron was significantly less methylated by an HF diet in the females (the same trend was observed in the males, but was not significant) (Fig. 7b). Genome-wide association studies have found SNPs in Galnt10 that are associated with BMI [48] and physical activity [49]. GALNT10 catalyzes the synthesis of mucin-type O-glycosylation, a type of post-translational modification. Important mucin-type O-linked glycoproteins include interluekin-2 and proteins involved in homing leukocytes to inflamed areas [50].

An HF diet was also associated with increased expression of the ladinin 1 ( $\operatorname{Lad} 1$ ) gene in the males and females, accompanied by decreased methylation in the females (with a non-significant trend in the males) at two adjacent DMRs in its first intron, which is also a promoter region (Fig. 7c). Lad1 is a part of the basement membrane, which increases around liver vessels in liver fibrosis. Basement membrane peptide levels increase in the serum as the severity of liver damage increases [51]. This is relevant because the HF mice had visibly fattier livers, in line with the increased risk of non-alcoholic fatty liver disease (NAFLD) due to obesity.

Further epigenetic evidence of liver distress induced by an HF diet is the upregulation of the collagen type I alpha 1 chain (Col1a1) gene in HF males and females, accompanied by increased methylation in the males at a DMR spanning exons 23 and 24 of the gene (Fig. $7 d$ ).
COL1A1 is a subunit of type 1 collagen, which accumulates in the liver during fibrosis and cirrhosis. When $\mathrm{Cal}-$ vente et al. [52] administered siRNA to degrade transcripts of Col1a1 in mice with advanced liver fibrosis, collagen deposition decreased by half and several other profibrogenic genes were downregulated. Our results support the notion that siRNA or other epigenetic treatments for elevated Col1a1 levels may help in obesity-related liver fibrosis.

The ATP binding cassette subfamily G member 5 (Abcg5) gene lies head-to-head with $A b c g 8$, and both were expressed higher in HF males than LF males. A DMR close to the start of both genes, located in the first intron of $A b c g 5$, had lower methylation due to an HF diet and may be involved in the co-regulation of the genes (Fig. $7 \mathrm{e})$. They encode proteins forming a heterodimer that facilitates the excretion of cholesterol into bile. Mutations in either gene are associated with atherosclerosis and sitosterolemia, a condition that leads to cardiovascular disease through the accumulation of sterols [53]. Our findings support previous studies that have identified the upregulation of $A b c g 5$ and $A b c g 8$ in response to insulin resistance and an HF diet [53-56]. The upregulation of the heterodimer may show an attempt to eliminate the excess cholesterol from the body, although HF mice still had 2-3 times as much serum cholesterol as LF mice.

\section{Discussion}

The results supported our hypothesis that a high-fat (HF) diet would alter the expression and methylation of genes involved in obesity and diabetes. The results also supported our hypothesis that the set of genes affected by an HF diet in males and females would not completely overlap. In the females, 2170 (5.6\%) of differentially methylated regions (DMRs) $(\mathrm{q}<0.05)$ occurred within genes that were differentially expressed due to diet, whereas in males 3209 (10.2\%) of DMRs did.

An HF diet was associated with drastic alterations in DNA methylation, gene expression, and physiology in the SM/J mice. By 17 weeks of age, mice on an HF diet weighed $70 \%$ more than mice on a low-fat (LF) diet, which we interpret as obesity. An HF diet significantly: increased all body weights and organ weights; decreased glucose and insulin tolerance; and increased serum levels of cholesterol, triglycerides, glucose, leptin, and insulin. The HF diet did not increase levels of free fatty acids in the serum, a trend that Do et al. [56] also found in C57BL/6 J mice, despite elevated fatty acid levels in the liver.

The expression of 4356 genes and the methylation of more than 7000 genes in the mouse liver were associated with diet. More than one-third of genes had at least one DMR associated with diet. The DMRs occurred in regulatory regions such as enhancers, transcription factor 
binding sites, and promoter flanking regions significantly more often than these regions occur in the genome, supporting the notion that methylation plays an important role in regulating the response to an HF diet. That role is not straightforward, however. The DMRs fell within differentially expressed genes significantly more often than expected by chance; however $31 \%$ of the DMRs did not even occur within genes at all, although $17 \%$ of those overlapped with enhancers or transcription factor binding sites. This is on par with the findings of Rönn et al. [21], who noted that fewer than $3 \%$ of adipose tissue DMRs following 6 months of exercise were located in differentially expressed genes in men. Methylation is only one piece of the puzzle; it will be interesting in the future to correlate the DNA methylation changes with histone modifications, as these two regulatory features work together to modulate gene expression.

Our gene expression findings mostly support those of other mouse studies, while highlighting differences that can be caused by factors such as genetic background, percent of fat in diet, and type of fat in the diet. For instance, Do et al. [56] compared liver expression of $\mathrm{HF}$ and $\mathrm{LF}$ male $\mathrm{C} 57 \mathrm{BL} / 6 \mathrm{~J}$ mice and found that an HF diet perturbed genes that were enriched for processes involved in immune and inflammatory response. Of the 332 genes they found differentially expressed due to an HF diet, 120 were the same as the ones we identified in the males (they did not study females). These included Adam11, $A b c g 5$, and $A b c g 8$, which we highlighted here as having differences in both expression and methylation associated with diet. We found 28 genes in common with Kim et al. [57], who identified 97 differentially expressed genes due to an $\mathrm{HF}$ diet in $\mathrm{C} 57 \mathrm{BL} / 6 \mathrm{~J}$ males. We found more genes in common with Kim et al. [57] and Do et al. [56] than either did with each other, even though they used the same mouse strain, which shows the utility of RNA-seq data over microarray data when comparing across studies. Of the 309 differentially expressed genes that Kirpich et al. [58] identified in male C57BL/6 mice due to an HF diet, we found 124 of the same genes. Kirpich et al. [58] shared 12 genes in common with Kim et al. [57] and 57 genes in common with Do et al. [56], and the only genes found in all three studies were Nsdhl and Sqle (Additional file 1: Figure S3). This highlights the difficulty of repeatability in gene expression studies. Like Inoue et al. [59] found in C57BL/6Ncrj male mice, we found that Pparg and its target gene Cd36 were both upregulated in the male and female HF mice, corroborating their conclusion that an HF diet induces liver steatosis by upregulating Pparg. Similar to other studies, we found an upregulation of genes in pathways associated with defense, stress, and inflammation responses [56, 57].

We compared our list of differentially expressed genes with those found in 9 other mouse strains exposed to an HF diet by Shockley et al. [60] and found 16-27 genes in common with the males in each study and 3-15 genes in common with the females (Additional file 1: Table S14). The strain dependent results underscore the importance of studying obesity in multiple strains of mice instead of basing conclusions off of one strain. Differences in duration of diet treatment [61], type of fat [62], and percent of fat in the diets [63] also can affect gene expression and may contribute to variation across studies.

Replicating DMRs across studies can be even more difficult than replicating gene expression, since methylation can be more variable and fewer studies have investigated it, especially genome-wide. Ge et al. [64] found that Lep had lower hepatic expression and higher promoter methylation in HF-fed female CD-1 mice. Here, we also found a hypermethylated DMR in the Lep promoter of the females $(q=0.02)$, but no difference in expression. Ge et al. [46] additionally found a hypomethylated Ppara promoter, and although we too found a DMR in Ppara, ours was hypermethylated in HF mice, it was located in the second intron, and the gene was not differentially expressed. Yoon et al. [65] identified hypomethylated $\mathrm{CpG}$ sites $1.5-\mathrm{kb}$ upstream of the Casp1 gene in C57BL/6 N male mice, but we found no DMRs there or in that gene. Like them, we did find lower expression of $N d u f b 9$ in HF males along with a DMR, but our DMR was hypomethylated by an HF diet whereas theirs was hypermethylated. As exemplified by this variability across studies, understanding the methylation changes underlying obesity will require much more research in the context of multiple genetic backgrounds.

Males and females responded differently to an HF diet. Compared to HF females, HF males had higher cholesterol, higher insulin, and higher glucose AUC during the intraperitoneal insulin tolerance test. The sex differences were visible on the levels of methylation and gene expression as well. There were more differentially expressed genes associated with diet in the males (3330) than in the females (1750), and more than 2000 genes were differentially expressed only in the males. The 184 genes with a significant sex-by-diet interaction were enriched for the epoxygenase P450 pathway, oxidation-reduction process, and response to stilbenoid, suggesting sex differences in these pathways mediate the difference between the male and female response to dietary fat. Cytochrome $\mathrm{P} 450$ genes are important for homeostasis and encode enzymes involved in metabolizing fatty acids and drugs, so sex differences in this pathway are relevant to pharmaceutical approaches to weight 
loss. Likewise, a sex-by-diet effect on the response to stilbenoids is interesting because they have been shown to regulate lipids, and Lin et al. [66] found that the stilbenoid TSG prevented NAFLD in HF-fed rats, with results that hinted at a small but inconclusive difference between males and females. Although there were more differentially expressed genes due to diet in males than in females, the opposite was true for DMRs. However, while there were fewer DMRs total in males, more of their DMRs occurred within genes that were differentially expressed due to diet.

\section{Conclusions}

This study identified thousands of genes that were differentially expressed and differentially methylated in response to a high-fat diet in SM/J mice. Genome-wide studies such as this are essential for developing a better understanding of the relevant epigenetic changes in obesity and identifying new targets for treatments. It is crucial that these treatments take sex into consideration, since-from the level of methylation to expression to obesity traits-males and females responded quite differently to an obesogenic diet.

\section{Additional file}

Additional file 1: Supplement. (ZIP $4328 \mathrm{~kb})$

\section{Acknowledgements}

We would like to acknowledge the peer reviewers for their invaluable feedback on an earlier draft.

\section{Funding}

The experimental work was funded in part by the American Heart Association under award number 16PRE26420105. The RNA sequencing was performed by GTAC, which is supported by grants from the $\mathrm{NCl}$ Cancer Center (P30 CA91842) and the National Center for Research Resources (UL1TR000448). The metabolomics was performed by the Core Laboratory for Clinical Studies and the Diabetes Models Phenotyping Core, which are supported by a grant from Washington University DRC (P30 DK020579). This publication is solely the responsibility of the authors and does not necessarily represent the official view of NCRR or NIH. The funding bodies had no role in: the design of the study; the collection, analysis, or interpretation of the data; or in writing the manuscript.

\section{Availability of data and materials}

The data is included in the manuscript and the supporting materials. The raw gene expression data is available on GEO (accession number GSE121525; https://www.ncbi.nlm.nih.gov/geo/query/acc.cgi?acc=GSE121525), and the raw methylation data is available on GEO (accession number GSE122016; https://www.ncbi.n/m.nih.gov/geo/query/acc.cgi?acc=GSE122016).

\section{Authors' contributions}

MRK and JMC are responsible for project planning. MRK, RZ, LH, and SS collected the mouse data. $\mathrm{XX}, \mathrm{DL}$, and TW performed the methylation sequencing and helped with the methylation analysis and interpretation. MRK, RZ, LH, and SS performed the data analysis. MRK wrote the manuscript, with invaluable feedback from JMC. JMC also provided funding and guidance. All authors have read and approved the manuscript.

\section{Ethics approval and consent to participate}

This study received ethics approval from Loyola University, followed an approved Institutional Animal Care and Use Committee protocol (Project \#1188).

\section{Consent for publication}

Not applicable.

Competing interests

The authors declare that they have no competing interests.

\section{Publisher's Note}

Springer Nature remains neutral with regard to jurisdictional claims in published maps and institutional affiliations.

\section{Author details}

${ }^{1}$ Department of Evolution, Ecology, and Population Biology, Washington University in St. Louis, St. Louis, MO 63105, USA. '2Department of Biology, Loyola University, Chicago, IL 60660, USA. ${ }^{3}$ Department of Genetics, Washington University in St. Louis, St. Louis, MO 63110, USA. ${ }^{4}$ Biology Department, 1032 W. Sheridan Road, Chicago, IL 60660, USA.

Received: 27 February 2018 Accepted: 28 November 2018

Published online: 07 December 2018

\section{References}

1. Skinner AC, Skelton JA. Prevalence and trends in obesity and severe obesity among children in the United States, 1999-2012. JAMA Pediatr. 2014; 168(6):561-6.

2. Lohman BJ, Gillette MT, Neppl TK. Harsh parenting and food insecurity in adolescence: the association with emerging adult obesity. J Adolesc Health. 2016;59(1):123-7.

3. Hagström H, Stål P, Hultcrantz R, Hemmingsson T, Andreasson A. Overweight in late adolescence predicts development of severe liver disease later in life: a 39years follow-up study. J Hepatol. 2016;65(2):363-8.

4. Finkelstein EA, Khavjou OA, Thompson H, Trogdon JG, Pan L, Sherry B, et al. Obesity and severe obesity forecasts through 2030. Am J Prev Med. 2012;42: 563-70.

5. Ogden $\mathrm{CL}$, Carroll MD, Kit BK, Flegal KM. Prevalence of childhood and adult obesity in the United States, 2011-2012. JAMA. 2014;311:806-14.

6. Yang L, Colditz GA. Prevalence of overweight and obesity in the United States, 2007-2012. JAMA Intern Med. 2015;1758:1412-3.

7. Cawley J, Meyerhoefer C. The medical care costs of obesity: an instrumental variables approach. J Health Econ. 2012;31:219-30.

8. Martinez JA. Body-weight regulation: causes of obesity. Proc Nutr Soc. 2000; 59:337-45.

9. Cheverud JM, Ehrich H, Hrbek T, Kenney JP, Pletscher LS, Semenkovich CF. Quantitative trait loci for obesity- and diabetes-related traits and their dietary responses to high-fat feeding in LGXSM recombinant inbred mouse strains. Diabetes. 2004;53:3328-36.

10. Ehrich TH, Hrbek T, Kenney-Hunt JP, Pletscher LS, Wang B, Semenkovich CF, et al. Fine-mapping gene-by-diet interactions on chromosome 13 in a LG/J x SM/J murine model of obesity. Diabetes. 2005;54:1863-72.

11. Bell CG, Walley AJ, Froguel P. The genetics of human obesity. Nat Rev Genet. 2005;6:221-34.

12. Pérusse L, Rankinen T, Zuberi A, Chagnon YC, Weisnagel SJ, Argyropoulos G, et al. The human obesity gene map: the 2004 update. Obes Res. 2005;13: 381-490.

13. O'Rahilly S, Farooqi IS. Genetics of obesity. Philos Trans R Soc Lond Ser B Biol Sci. 2006;361:1095-105.

14. Mau T, Yung R. Potential of epigenetic therapies in non-cancerous conditions. Front Genet. 2014;5:438

15. Campión J, Milagro Fl, Martínez JA. Individuality and epigenetics in obesity. Obes Rev. 2009:10:383-92.

16. Emilsson $\mathrm{V}$, Thorleifsson G, Zhang B, Leonardson AS, Zink F, Zhu J, et al. Genetics of gene expression and its effect on disease. Nature. 2008;452: 423-8.

17. Bird A. Perceptions of epigenetics. Nature. 2007:447:396-8.

18. Razin A, Cedar H. DNA methylation and gene expression. Microbiol Rev. 1991:5:451-8 
19. Barua S, Kuizon S, Chadman KK, Flory MJ, Brown W, Junaid MA. Single-base resolution of mouse offspring brain methylome reveals epigenome modifications caused by gestational folic acid. Epigenetics Chromatin. 2014;7:3.

20. Lomba A, Milagro Fl, García-Díaz DF, Marti A, Campión J, Ma JA. Obesity induced by a pair-fed high fat sucrose diet: methylation and expression pattern of genes related to energy homeostasis. Lipids Health Dis. 2010;9:60.

21. Rönn $T$, Volkov $P$, Davegårdh $C$, Dayeh $T$, Hall E, Olsson AH, et al. A six months exercise intervention influences the genome-wide DNA methylation pattern in human adipose tissue. PLoS Genet. 2013;9:e1003572.

22. Malodobra-Mazur MA, Dziewulska A, Kozinski K, Dobrzyn P, Kolczynska K, Janikiewicz J, et al. Stearoyl-CoA desaturase regulates inflammatory gene expression by changing DNA methylation level in 3T3 adipocytes. Int J Biochem Cell Biol. 2014;55:40-50

23. van Dijk SJ, Molloy PL, Varinli H, Morrison JL, Muhlhausler BS, Members of EpiSCOPE. Epigenetics and human obesity. Int J Obes. 2015;39:85-97.

24. Baumeier $C$, Saussenthaler $S$, Kammel A, Jähnert M, Schlüter L, Hesse D, et al. Hepatic DPP4 DNA methylation associates with fatty liver. Diabetes. 2017:66:25-35.

25. Zwamborn RA, Slieker RC, Mulder PC, Zoetemelk I, Verschuren L, Suchiman HE, Toet KH, Droog S, Slagboom PE, Kooistra T, Kleemann R, Heijmans BT. Prolonged high-fat diet induces gradual and fat depot-specific DNA methylation changes in adult mice. Sci Rep. 2017;7:43261.

26. Montgomery MK, Hallahan NL, Brown SH, Liu M, Mitchell TW, Cooney GJ, Turner N. Mouse strain-dependent variation in obesity and glucose homeostasis in response to high-fat feeding. Diabetologia. 2013;56(5):1129-39.

27. Andrikopoulos S, Massa CM, Aston-Mourney K, Funkat A, Fam BC, Hull RL, Kahn SE, Proietto J. Differential effect of inbred mouse strain (C57BL/6, DBA $2,129 T 2)$ on insulin secretory function in response to a high fat diet. J Endocrinol. 2005;187(1):45-53.

28. Widiker S, Karst S, Wagener A, Brockmann GA. High-fat diet leads to a decreased methylation of the Mc4r gene in the obese BFMl and the lean B6 mouse lines. J Appl Genet. 2010;51:193-7.

29. Cheverud JM, Pletscher LS, Vaughn TT, Marshall B. Differential response to dietary fat in large (LG/J) and small $(\mathrm{SM} / \mathrm{J})$ inbred mouse strains. Physiol Genomics. 1999:15:33-9.

30. Ehrich TH, Kenney JP, Vaughn TT, Pletscher LS, Cheverud JM. Diet, obesity, and hyperglycemia in LG/J and SM/J mice. Obes Res. 2003;11:1400-10.

31. Partridge C, Fawcett GL, Wang B, Semenkovich CF, Cheverud JM. The effect of dietary fat intake on hepatic gene expression in LG/J and SM/J mice. BMC Genomics. 2014;15:99.

32. MacArthur J. Genetics of body size and related characters. I. Selection of small and large races of the laboratory mouse. Amer Natur. 1944;78:142-57.

33. Lam YY, Ha CW, Campbell CR, Mitchell AJ, Dinudom A, Oscarsson J, Cook DI, Hunt NH, Caterson ID, Holmes AJ, Storlien LH. Increased gut permeability and microbiota change associate with mesenteric fat inflammation and metabolic dysfunction in diet-induced obese mice. PLoS One. 2012;7(3): e34233.

34. Le Floch J-P, Escuyer P, Baudin E, Baudon D, Perlemuter L. Blood glucose area under the curve: methodological aspects. Diabetes Care. 1990;13(2):172-5.

35. Cheverud JM, Lawson HA, Fawcett GL, Wang B, Pletscher LS, Fox AR, et al. Diet-dependent genetic and genomic imprinting effects on obesity in mice. Obesity. 2011;19(1):160-70.

36. Dobin A, Davis CA, Schlesinger F, Drenkow J, Zaleski C, Jha S, et al. STAR: ultrafast universal RNA-seq aligner. Bioinformatics. 2013;29:15-21.

37. Robinson MD, McCarthy DJ, Smyth GK. edgeR: a Bioconductor package for differential expression analysis of digital gene expression data. Bioinformatics. 2010;26:139-40.

38. Luo W, Friedman M, Shedden K, Hankenson K, Woolf P. GAGE: generally applicable gene set enrichment for pathway analysis. BMC Bioinformatics. 2009;10:161.

39. Luo W, Brouwer C. Pathview: an R/Bioconductor package for pathway-based data integration and visualization. Bioinformatics. 2013;29:1830-1.

40. Li D, Zhang B, Xing X, Wang T. Combining MeDIP-seg and MRE-seg to investigate genome-wide CpG methylation. Methods. 2015;72:29-40.

41. Stevens $M$, Cheng JB, Li D, Xie M, Hong C, Maire CL, et al. Estimating absolute methylation levels at single CPG resolution from methylation enrichment and restriction enzyme sequencing methods. Genome Res. 2013;23:1541-53.

42. Zhang B, Zhou Y, Lin N, Lowdon RF, Hong C, Nagarajan RP, et al. Functional DNA methylation differences between tissues, cell types, and across individuals discovered using the M\&M algorithm. Genome Res. 2013;23: 1522-40.

43. Fisher RA. Statistical methods for research workers. 12th ed. Edinburgh: Oliver \& Boyd; 1954. p. 356

44. Benjamini Y, Hochberg Y. Controlling the false discovery rate: a practical and powerful approach to multiple testing. J R Stat Soc Ser B Methodol. 1995;57:289-300.

45. Yates A, Akanni W, Ridwan Amode M, Barrell D, Billis K, Carvalho-Silva D, et al. Ensembl 2016. Nucleic Acids Res. 2016;44:D710-6.

46. Ziller MJ, Hansen KD, Meissner A, Aryee MJ. Coverage recommendations for methylation analysis by whole genome bisulfite sequencing. Nat Methods. 2015;12(3):230-2.

47. Takahashi E, Sagane K, Nagasu T, Kuromitsu J. Altered nociceptive response in ADAM11-deficient mice. Brain Res. 2006;1097:39-42.

48. Monda KL, Chen GK, Taylor KC, Palmer C, Edwards TL, Lange LA, et al. A meta-analysis identifies new loci associated with body mass index in individuals of African ancestry. Nat Genet. 2013;45:690-6.

49. Ahmad S, Zhao W, Renström F, Rasheed A, Samuel M, Zaidi M, et al. Physical activity, smoking, and genetic predisposition to obesity in people from Pakistan: the PROMIS study. BMC Med Genet. 2015;16:114.

50. Hang HC, Bertozzi CR. The chemistry and biology of mucin-type O-linked glycosylation. Bioorg Med Chem. 2005;13:5021-34.

51. Walsh KM, Fletcher A, MacSween RN, Morris AJ. Basement membrane peptides as markers of liver disease in chronic hepatitis C. J Hepatol. 2000;32:325-30.

52. Calvente CJ, Sehgal A, Popov Y, Kim YO, Zevallos V, Sahin U, et al. Specific hepatic delivery of procollagen a1(I) small interfering RNA in lipid-like nanoparticles resolves liver fibrosis. Hepatology. 2015;62:1285-97.

53. Yu XH, Qian K, Jiang N, Zheng XL, Cayabyab FS, Tang CK. ABCG5/ABCG8 in cholesterol excretion and atherosclerosis. Clin Chim Acta. 2014;428:82-8.

54. Biddinger SB, Haas JT, Yu BB, Bezy O, Jing E, Zhang W, et al. Hepatic insulin resistance directly promotes formation of cholesterol gallstones. Nat Med. 2008;14:778-82.

55. Yamazaki $Y$, Hashizume $T$, Morioka H, Sadamitsu S, Ikari A, Miwa M, et al. Diet-induced lipid accumulation in liver enhances ATP-binding cassette transporter g5/g8 expression in bile canaliculi. Drug Metab Pharmacokinet. 2011;26:442-50.

56. Do G, Oh HY, Kwon EY, Cho YY, Shin SK, Park HJ, et al. Long-term adaptation of global transcription and metabolism in the liver of high-fat diet-fed C57BL/6J mice. Mol Nutr Food Res. 2011;55:S173-85.

57. Kim S, Hashizume T, Morioka H, Sadamitsu S, Ikari A, Miwa M, et al. Hepatic gene expression profiles in a long-term high-fat diet-induced obesity mouse model. Gene. 2004;340:99-109.

58. Kirpich IA, Gobejishvili LN, Bon Homme M, Waigel S, Cave M, Arteel G, et al Integrated hepatic transcriptome and proteome analysis of mice with high-fat diet-induced nonalcoholic fatty liver disease. J Nutr Biochem. 2011;22:38-45.

59. Inoue M, Ohtake T, Motomura W, Takahashi N, Hosoki Y, Miyoshi S, et al. Increased expression of PPARgamma in high fat diet-induced liver steatosis in mice. Biochem Biophys Res Commun. 2005;336:215-22.

60. Shockley KR, Witmer D, Burgess-Herbert SL, Paigen B, Churchill GA. Effects of atherogenic diet on hepatic gene expression across mouse strains. Physiol Genomics. 2009;39:172-82

61. Almon RR, Dubois DC, Sukumaran S, Wang X, Xue B, Nie J, Jusko WJ. Effects of high fat feeding on liver gene expression in diabetic goto-kakizaki rats. Gene Regul Syst Bio. 2012;6:151-68.

62. Timmers S, de Vogel-van den Bosch J, de Wit N, Schaart G, van Beurden D, Hesselink M, van der Meer R, Schrauwen P. Differential effects of saturated versus unsaturated dietary fatty acids on weight gain and myocellular lipid profiles in mice. Nutr Diabetes. 2011;1:e11.

63. Tanaka M, Akihito Y, Shimizu M, Saito Y, Kumakura K, Asakura T, Nagai T. Transcriptomic responses of the liver and adipose tissues to altered carbohydrate-fat ratio in diet: an isoenergetic study in young rats. Genes Nutr. 2017;12:10

64. Ge Z-J, Luo S-M, Lin F, Liang Q-X, Huang L, Wei Y-C, et al. DNA methylation in oocytes and liver of female mice and their offspring: effects of high-fatdiet-induced obesity. Environ Health Perspect. 2014;122:159-64.

65. Yoon A, Tammen SA, Park S, Han SN, Choi S-W. Genome-wide hepatic DNA methylation changes in high-fat diet-induced obese mice. Nutr Res Pract. 2017:11:105-13.

66. Lin P, Lu J, Wang Y, Gu W, Yu J, Zhao R. Naturally occurring stilbenoid TSG reverses non-alcoholic fatty liver diseases via gut-liver axis. PLoS One. 2015; 10:e0140346. 